\title{
Finite temperature spectral functions in the $O(N)$ model
}

\author{
Jan M. Pawlowski, ${ }^{1,2}$ Nils Strodthoff, ${ }^{3}$ and Nicolas Wink ${ }^{1}$ \\ ${ }^{1}$ Institut für Theoretische Physik, Universität Heidelberg, \\ Philosophenweg 16, 69120 Heidelberg, Germany \\ ${ }^{2}$ ExtreMe Matter Institute EMMI, GSI, Planckstrasse 1, D-64291 Darmstadt, Germany \\ ${ }^{3}$ Nuclear Science Division, Lawrence Berkeley National Laboratory, Berkeley, California 94720, USA
}

(Received 4 December 2017; published 9 October 2018)

\begin{abstract}
We directly calculate spectral functions in the $O(N)$ model at finite temperature within the framework of the functional renormalization group. Special emphasis is put on a fully numerical framework involving four-dimensional regulators preserving Euclidean $O(4)$ and Minkowski Lorentz invariance, an important prerequisite for future applications. Pion and sigma meson spectral functions are calculated for a wide range of temperatures across the phase transition illustrating the applicability of the general framework for finite temperature applications. In addition, various aspects concerning the interplay between the Euclidean and real-time two-point function are discussed.
\end{abstract}

DOI: 10.1103/PhysRevD.98.074008

\section{INTRODUCTION}

The access to real-time correlation functions is key to the theoretical understanding of many interesting physics phenomena ranging from the bound-state spectrum of the theory at hand over decays to the dynamical evolution of both close-to- and far-from-equilibrium systems. However, strongly correlated systems are typically only accessible within numerical computations with either lattice or functional approaches. These approaches have been mostly used in their Wick rotated form in Euclidean space if it comes to applications to strongly correlated systems in higher dimensions such as QCD.

In the present work, we report on the progress within a fully numerical approach to directly compute real-time correlation functions introduced in [1,2]. This formalism is based on the functional renormalization group (FRG) [3], a suitable nonperturbative method; see [4-12] for QCDrelated reviews. Such a numerical framework is required for application to strongly correlated systems whose degrees of freedom have a nontrivial dispersion relation. Furthermore, already in Euclidean applications the use of regulators preserving Euclidean $O(4)$ and Minkowski Lorentz invariance is crucial in situations with finite temperatures and density. We would also like to emphasize that the current real-time approach is useful and applicable to finite density situations in Euclidean space: The $O(4)$-invariant

Published by the American Physical Society under the terms of the Creative Commons Attribution 4.0 International license. Further distribution of this work must maintain attribution to the author(s) and the published article's title, journal citation, and DOI. Funded by SCOAP. regulators and methods discussed here allow us to guarantee the silver-blaze property without violating Lorentz symmetry. Moreover, the flow is local in both momentum and frequency space. It is this combination of properties which is crucial for a quantitative determination of the density. The above requirements are met in QCD at finite temperature and density but also in ultracold atomic systems. Both systems are key application areas of the current real-time approach.

FRG approaches to real-time computations close to the present one have been set up in $[13,14]$. While the former one shares the space-time symmetry with the present approach, the latter one shares the locality in momentum space. The present approach combines both momentum and frequency locality as well as Lorentz invariance.

Real-time applications range from the dynamics of low-dimensional systems to the description of farfrom-equilibrium dynamics; for a selection of works, see [15-35]. Most of the implementations have in common that either they are not directly applicable to higher-dimensional systems, or one would have to rederive the plethora of Euclidean results already obtained in the Euclidean version of the FRG. This situation asks for an approach which allows us to either make direct use of available Euclidean results or at least be able to benchmark the real-time results with the Euclidean ones already obtained. In addition, the physical content of Euclidean truncations in terms of scattering processes, resonances, particles, and decay channels can be directly accessed from the real-time correlation functions and identified with the equivalent Euclidean part. In principle, it is possible to solve the hard problem of obtaining real-time correlation functions from reconstruction of Euclidean data. This task requires a very 
good resolution of the momentum and frequency dependence of the Euclidean correlation functions; for related FRG work, see [36-48]. Subsequently, applied methods based on Bayesian reconstruction like the maximum entropy method [49-54] are able to produce remarkable results in many cases. For spectral reconstructions based on Euclidean FRG data, see, e.g., [55-58]. Unfortunately, these methods are mathematically not guaranteed to converge towards the correct correlation function. Other methods which do not have this problem [59-61] suffer from sign problems and require unachievable numerical precision for reliable results. All of these obstacles can be overcome by a direct numerical calculation.

Our long-term goals are the hadron spectrum, transport coefficients, and other real-time observables within the framework of the fQCD Collaboration [62], which aims at a quantitative first-principle description of QCD from the FRG. This stresses once more the importance of a fully numerical approach due to the sophisticated technical level already required at the Euclidean level to obtain quantitatively competitive results [43,45-47]. This work is a necessary piece in order to close the gap between the calculation of Euclidean correlation functions and dynamical observables such as transport coefficients. These quantities can be obtained conveniently from the spectral functions as demonstrated with the shear viscosity [56,57] from reconstructed spectral functions.

\section{II. $O(N)$ MODEL SPECTRAL FUNCTIONS AT FINITE TEMPERATURE}

The classical action of the $O(N)$ model is given by

$$
S=\int \mathrm{d}^{4} x\left(\frac{1}{2}\left(\partial_{\mu} \phi_{a}\right)^{2}+\frac{1}{2} m \phi_{a}^{2}+\frac{\lambda}{4}\left(\phi_{a}^{2}\right)^{2}\right),
$$

with an $\mathrm{N}$ component scalar field $\phi_{a}$, the mass $m$, and the coupling $\lambda$. As mentioned before, we are using the FRG to get access to the quantum effective action $\Gamma$. The scaledependent effective action obeys a one-loop exact flow, the Wetterich equation [3],

$$
\partial_{t} \Gamma_{k}=\frac{1}{2} \operatorname{Tr}\left\{\left(\Gamma_{k}^{(2)}+R_{k}\right)^{-1} \partial_{t} R_{k}\right\} .
$$

The regulator $R_{k}$ suppresses momenta lower than the RG scale $k$, while its scale derivative $t=\log k$ servers as an UV regulation. The inverse quantity appearing in (2) is the regularized propagator related to the scale-dependent effective action via functional derivatives. The initial condition for (2) at asymptotically large infrared cutoff scales $k$ is the bare classical or UV-relevant action. In turn, at vanishing cutoff scale, $\Gamma_{k=0}$ is the full quantum effective action; for more introductory details, see [4-12].

The calculation of real-time observables such as spectral functions starting from a Euclidean formalism represents a difficult problem, as it requires the analytic continuation from Euclidean to Minkowski signature. In this work, we follow the formalism put forward in [1], where the analytic continuation is carried out on the level of the equation itself. Unfortunately, the straightforward evaluation of the Euclidean correlation function for complex momenta does not lead to the desired retarded correlation functions required for the extraction of the spectral function. The procedure in [1] thus requires a deformation of the integration contour into the complex plane with appropriate corrections in order to recover the desired real-time observable. The following discussion is restricted to the simplified case referred to as propagator approximation in the following, where only the Minkowski-momentum dependence of propagators is taken into account. This approximation obviously misses bound-state effects in $n$ point vertices that are hidden in a nontrivial Minkowskimomentum dependence of the vertices. However, such effects could still be captured by a dynamical hadronization of the corresponding channels in the respective vertices within this approximation.

Here we present a formal solution which holds in the propagator approximation irrespective of the analytic structure of the propagators under consideration. Following [1], we start by recapitulating the case of vanishing temperature. In this approximation, it is sufficient to consider a single diagram topology, namely, the self-energy contribution to the two-point function involving two three-point vertices. Here we are interested in contributions to the retarded twopoint functions, as the latter relates directly to the spectral function. Given analytical propagators as a function of a complex momentum variable, it turns out that the contribution to the retarded correlation function is obtained by integration along a particular path in the complex momentum plane. The defining property of this path is that it represents a continuous deformation of the integration path along the real axis for vanishing Minkowski external momentum. This is illustrated in Fig. 1(a) for the case of two propagators which only carry complex structures on the Minkowski axis, but this argument generalizes straightforwardly to propagators with a general complex structure. In the case of only simple poles, the contribution arising from integrating along the simple contour $\mathcal{C}_{\text {eucl }}$ relates to the desired contribution to the retarded correlator obtained by integrating along $\mathcal{C}_{\text {ret }}$ by a closed contour integration. The difference between the two results can be written as a sum of residues of certain poles in the complex plane, which is exactly the situation covered in [1].

At finite temperature, it turns out that the simple evaluation of the Matsubara sum in the analytically continued expression does again not lead to the desired contribution to the self-energy of the retarded propagator. As demonstrated in [1] for a propagator with only simple poles, the difference between the two results can be written as a sum of residues corresponding to all poles of the 


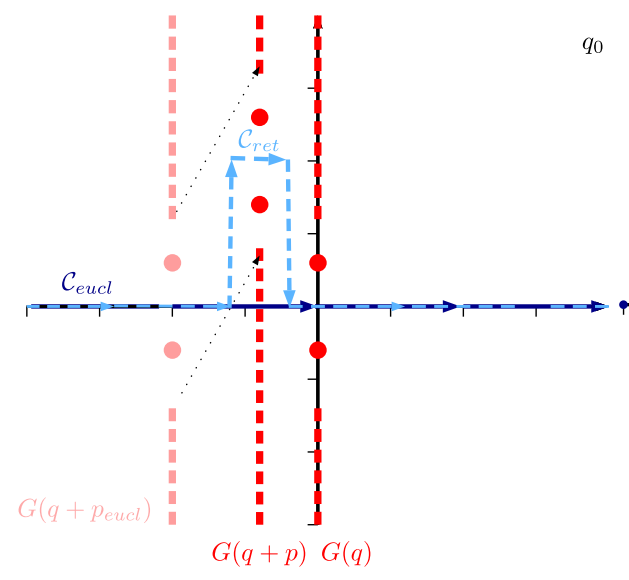

(a) Vacuum

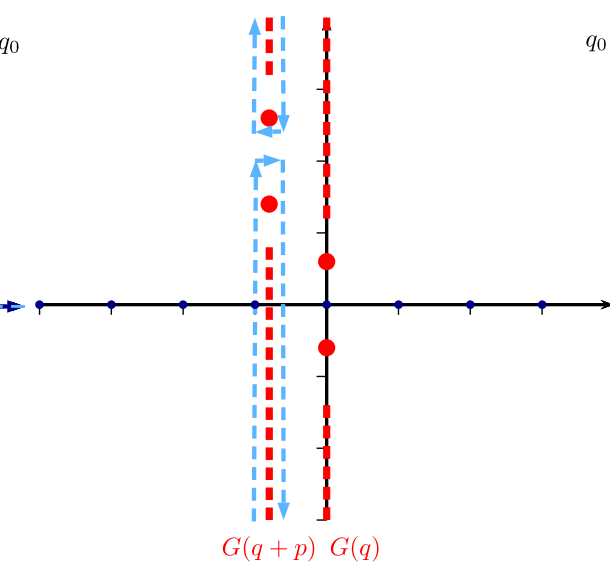

(b) Finite temperature

FIG. 1. The analytic structure of a product of two propagators, one of which is shifted by an external momentum $p$. Red colors show poles and branch cuts from different propagators, while the corresponding integration contours are shown in blue. In the vacuum case (Fig. 1(a)), the analytic continuation corresponds to a simple deformation of the contour, $\mathcal{C}_{\text {eucl }} \rightarrow \mathcal{C}_{\text {ret }}$. At finite temperature (Fig. 1(b)), one can correct the mistake from the Matsubara sum (dark blue dots) by calculating the correction from an integration along the light blue path. More details can be found in the main text.

propagator involving the external frequency weighted by a difference of thermal occupation numbers. This generalizes straightforwardly to the situation with propagators with a general complex structure: The correction term is obtained by evaluating contour integrals surrounding all complex structures of the propagator involving the external frequency weighted with a difference of occupation numbers $n\left(\mathrm{i}\left(p_{0}+q_{0}\right)\right)-n\left(\mathrm{i} q_{0}\right)$. This is illustrated in Fig. 1(b). This procedure represents a formal solution of the analytical continuation problem in the propagator approximation. The numerical implementation of this procedure turns out to be very challenging, as it requires one to evaluate contour integrals in the close vicinity of first- and secondorder poles [as soon as the regulator term $G \dot{R} G(q)$ is introduced].

The above discussion applies to generic field theories. For the explicit computations in this work, we restrict ourselves to an $O(N)$ model. This model has been studied in great detail with the FRG; see the reviews [4-12] and references therein. Here we study the model at finite temperature with a scale-dependent effective potential and scale-dependent dispersion. In this case, the propagators show only simple poles, and the correction term can be evaluated as a sum of residue contributions instead of an actual contour integration; further details can be found in Appendix C.

Technically, the retarded two-point correlator is obtained from the Euclidean correlation function [cf. (2)], which is the inverse of the second derivative of the quantum effective action $\Gamma[\phi]$ with respect to the field

$$
\Gamma_{R}^{(2)}(\omega,|\vec{p}|)=\lim _{\varepsilon \rightarrow 0} \Gamma_{\text {Eucl }}^{(2)}\left(p_{0}=-\mathrm{i}(\omega+\mathrm{i} \varepsilon), \mid \vec{p}\right) \mid,
$$

which depends on the frequency $\omega$ and the three momentum $|\vec{p}|$. From the retarded propagator, the inverse twopoint function, one can then obtain the spectral function

$$
\rho(\omega, \vec{p})=2 \operatorname{Im} G_{R}(\omega, \vec{p}) Z(0),
$$

where $Z(0)$ is the wave-function renormalization at vanishing momentum. The spectral function in (4) is renormalization group invariant, but it is not normalized to one,

$$
\int_{\mathbb{R}^{+}} d \omega^{2} \rho(\omega, 0)=\mathcal{N}_{\rho}
$$

This comes from the normalization with $Z(0)$. Typically, the physical spectral function is normalized to one,

$$
\hat{\rho}=\frac{1}{\mathcal{N}_{\rho}} \rho
$$

\section{A. Truncations}

In order to meet the requirement of only having to take pole corrections into account when calculating spectral functions, we restrict our effective action to the following form

$$
\Gamma_{k}=\int \mathrm{d}^{4} x\left(\frac{Z}{2}\left(\partial_{\mu} \phi_{a}\right)^{2}+\frac{Y}{8}\left(\partial_{\mu} \rho\right)^{2}+V(\sigma)\right),
$$

with the mesonic field $\phi=(\sigma, \vec{\pi})$. In (7) and in the following, we suppress the scale dependence on $k$ on the right-hand side in the wave-function renormalization $Z_{k}$ and the coupling $Y_{k}$ as well as the effective potential $V_{k}$. The effective potential $V(\sigma)$ is split into a part only 
depending on the $O(4)$-invariant $\rho=\frac{1}{2}\left(\pi_{a}^{2}+\sigma^{2}\right)$ and a part explicitly breaking the symmetry, thus, allowing for the Goldstone bosons to acquire a finite mass

$$
V(\sigma)=U(\rho)-c \sigma
$$

Note that the linear breaking term drops out of all dynamical equations, and in particular the flow equations. While this fact is hidden in an expansion about the flowing minimum, it is apparent within an expansion around a fixed expansion point in the bare field $\sigma$; see [63]. In the present work, we choose the latter expansion for both stability and for a self-consistent frequency dependence; see [64]. We choose the expansion point close but above the IR minimum $\langle\sigma\rangle$, as argued in [63]. We take into account terms up to $\phi^{14}$, and the convergence of the results has been checked.

The truncation (7) is referred to as LPA' $+Y$, and we additionally consider a second truncation with $Z=1$, $Y=0$ corresponding to the usual LPA (local potential approximation) scheme. In the following, we also drop the contribution to the four-point vertex from the $Y$ term. Then, the two dressing functions $Z$ and $Y$ can effectively be reabsorbed into a dressing for the pion $Z_{\pi}$, and one for the sigma meson $Z_{\sigma}$. Finite temperature is introduced via the Matsubara formalism in the usual manner. As argued in [1], we are mostly concerned with regulators depending on the Lorentz-invariant momentum configurations $q^{2}$ and is discussed at large in said reference. Further details about the regulator in the current work are given in Appendix A, and a comparison with a Lorentz invariance breaking regulator is discussed in Appendix B.

\section{RESULTS}

We first display the temperature and momentum dependence of the spectral functions. Furthermore, we extract the pole mass and compare it to the curvature mass. Details about the numerical implementation are given in Appendix C.

\section{A. Spectral functions at vanishing external momentum}

Here we show the temperature evolution of the pion and sigma spectral function in the LPA' $+\mathrm{Y}$ approximation. The spectral functions feature several district structures with a clear physical interpretation; see, e.g., [65] for a detailed discussion of the different processes in the quarkmeson model. In general, there are two different cut structures at finite temperature and vanishing external momentum in the propagator extended to the complex plane. The unitarity cut spans from the multiparticle decay threshold to infinity, i.e., $\omega \in\left[\mu_{\text {thresh }}, \infty\right)$, which is present in any interacting theory. Furthermore, the Landau cut is present at smaller frequencies, which is purely medium dependent and gives rise to inverse scattering processes [66] with the heat bath. These scattering processes give rise to Landau damping, hence, the name. Finally, delta functions represent stable particles. Our results for the spectral functions at vanishing external momentum in the LPA and LPA' $+\mathrm{Y}$ truncation are depicted in Fig. 2. For the sigma meson, there are two different processes available, i.e., $\sigma^{*} \rightarrow \pi+\pi$ and $\sigma^{*} \rightarrow \sigma+\sigma$, and no Landau cut structure. While for the pion, we have $\pi^{*} \rightarrow \pi+\sigma$ for the unitarity cut and $\pi^{*}+\pi \rightarrow \sigma$ for the Landau cut. While it might seem that the Landau cut vanishes for very small frequencies, we would like to remark that a finite value is possible in principle. However, it will be exponentially suppressed since the contributions are proportional to occupation numbers. Additionally, Fig. 2 suggests that the Landau cut only has support up to a finite frequency and vanishes below. At vanishing temperature, we have the expected stable pion and no stable sigma particle. At finite temperature, the sigma meson emerges as a stable particle as $O(4)$ symmetry gets restored. The presence of stable particles at finite temperatures is an artifact of the current truncation.

The difference between the two truncations is most prominently seen at multiple particle decay thresholds, which involve a sigma meson demonstrating the effect of the wave-function renormalization shown in Fig. 4(c) on the curvature mass, as in both truncations the proper pole mass is not coupled back into the system. A problem that is numerically not traceable in the current formalism; c.f. the discussion in Appendix C. The cut structure of propagators is best seen in the imaginary part of the two-point function $\operatorname{Im} \Gamma^{(2)}(\omega)$, as a finite value translates directly into a cut of the propagator; the result is shown in Fig. 3.

The finite part at small frequencies that vanishes for larger temperatures in the pion shows again the Landau cut. For larger frequencies, the unitarity cut shows clear decay thresholds; i.e., in the sigma meson, the different thresholds, and their degeneracy for high temperatures can be seen nicely.

Our results compare qualitatively well to the results obtained using a spatially flat regulator (cf. the discussion in Appendix B) in the quark-meson model [67-70].

\section{B. Pole, screening, and curvature masses}

It is interesting to compare the real-time pole and screening masses with the Euclidean curvature mass; for a detailed discussion of the respective definitions in the present FRG context, see, e.g., [64]. The curvature mass is typically used in Euclidean computations within low energy effective field theories for QCD. There, the physical pion and sigma masses are input parameters and are identified with the respective curvature masses. However, they have to be identified with the pole mass, and, hence, we have to check how well such an identification works.

In the case of a stable particle, the position of the pole can be directly extracted from the spectral function. For particles with a finite decay width, this is not possible 

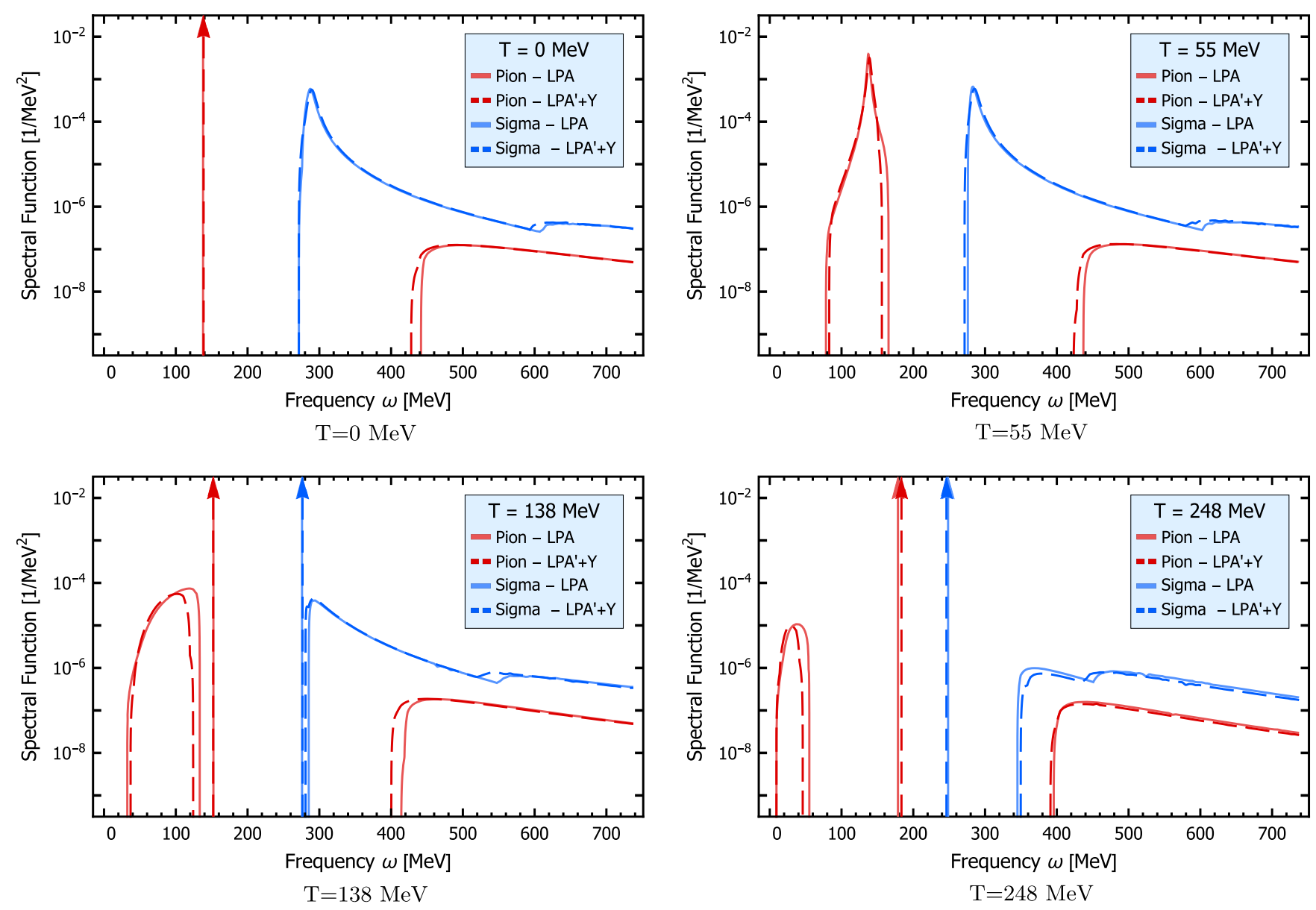

FIG. 2. Spectral functions at vanishing three momentum for different temperatures comparing LPA to LPA' + Y.

anymore, as the pole leaves the physical sheet of the complex energy plane and is found on the second Riemann sheet. On the other hand, the pole mass $m_{\text {pole }}=1 / \xi_{t}$ is the inverse temporal screening length, which can be extracted from

$$
\lim _{t \rightarrow \infty} G_{E}(t, 0) \propto e^{-t / \xi_{t}}
$$

Furthermore, the screening mass is $m_{\text {screen }}=1 / \xi_{\text {spat }}$, that is the inverse spatial screening length,

$$
\lim _{|\vec{x}| \rightarrow \infty} G_{E}(0, \vec{x}) \propto e^{-|\vec{x}| / \xi_{\text {spatial }}},
$$

and the curvature mass is

$$
m_{\mathrm{cur}}^{2}=\lim _{\vec{p} \rightarrow 0} \frac{\Gamma_{E}^{(2)}(0, \vec{p})}{Z(0, \vec{p})} .
$$

Note that the wave-function renormalization $Z$ in (11) ensures the RG invariance of the latter. However, the necessity of a choice of the momentum configuration [in (11), it is $p_{0}=0, \vec{p} \rightarrow 0$ ] introduces a scheme dependence, while the pole and screening masses are scheme independent.
Note also that at finite temperature, the order of limits in (11) matter, as the two limits do not commute anymore

$$
\lim _{\vec{p} \rightarrow 0} \lim _{p_{0} \rightarrow 0} \Gamma^{(2)}\left(p_{0}, \vec{p}\right) \neq \lim _{p_{0} \rightarrow 0} \lim _{\vec{p} \rightarrow 0} \Gamma^{(2)}\left(p_{0}, \vec{p}\right),
$$

where the order of limits on the lhs is referred to as static and the limit on the rhs as plasmon; for details, see, e.g., [71]. If not stated otherwise, $Z(0,0)$ is understood in the static limit. The difference between the two limits can be seen easily in the corresponding spectral functions, as the static limit contains the transport peak, while this structure is absent in the plasmon limit; compare, e.g., Fig. 2 with Fig. 6, and see the corresponding discussion in Sec. III C.

The difference between the two limits can also be seen in the corresponding Euclidean correlation functions shown in Fig. 4(b), where the green line shows the propagator as a function of the external momentum at the zeroth Matsubara mode, i.e., approaching the static limit. The red line shows the propagator as a function of the external Euclidean frequency, continuously defined due to the analytic continuation involved, which approaches the plasmon limit. As soon as a small external 

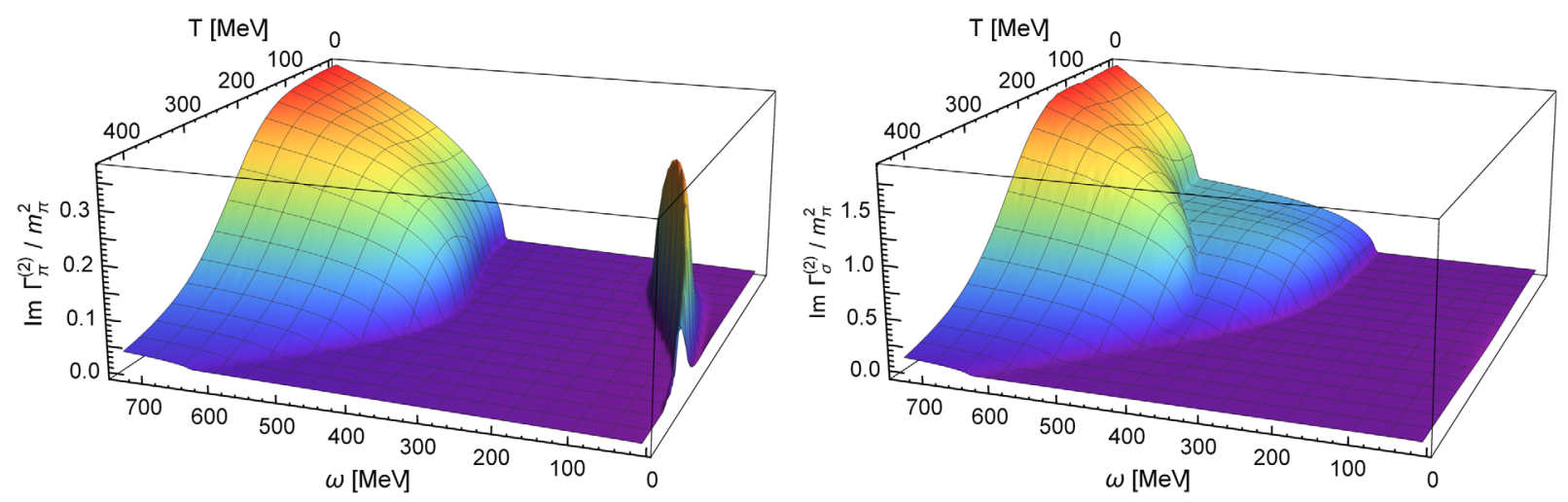

FIG. 3. Cut structure of the propagators in the frequency-temperature plane.

momentum is introduced, the static limit is implicitly taken, and the scale is given by the external momentum shown as vertical lines. For better visibility, the trivial mismatch for larger $\vec{p}$ has been subtracted, $\tilde{\Gamma}^{(2)}\left(p_{0}, \vec{p}\right)=$ $\Gamma^{(2)}\left(p_{0}, \vec{p}\right)-\vec{p}^{2}$ and $\tilde{G}=\tilde{\Gamma}^{-1}$.

We can now use (11) together with (13) together with the spectral representation of the Euclidean propagator

$$
Z(0,0) G_{E}\left(p_{0}, \vec{p}\right)=\int \mathrm{d} \eta \frac{\rho(\eta, \vec{p})}{\eta+\mathrm{i} p_{0}}
$$

to derive a relation between the curvature mass and the spectral representation

$$
m_{\mathrm{cur}}^{2}=\frac{\int \frac{\mathrm{d} \eta}{\eta} \hat{\rho}(\eta, 0)}{\int \frac{\mathrm{d} \eta}{\eta} \frac{1}{\eta^{2}} \hat{\rho}(\eta, 0)},
$$

with the normalised spectral function (6). In case a stable particle is present, the spectral function can be split in two positive parts as follows:

$$
\begin{aligned}
\hat{\rho} & =\hat{\rho}_{\text {pole }}+\hat{\rho}_{\text {cut }}, \\
\hat{\rho}_{\text {pole }} & =\operatorname{sign}(\omega) Z_{\text {pole }} \delta\left(p^{2}-m_{\text {pole }}^{2}\right) .
\end{aligned}
$$

The normalization of the pole is smaller than one: $Z_{\text {pole }}=$ $1-\int d \omega^{2} \hat{\rho}_{\text {cut }}<1$, which follows from the normalization of the spectral function and the positivity of $\rho_{\text {cut }}$. Using this split in (13), we arrive at

$$
m_{\text {cur }}^{2}=m_{\text {pole }}^{2} \frac{Z_{\text {pole }}+m_{\text {pole }}^{2} \int \frac{\mathrm{d} \eta}{\eta} \rho_{\text {cut }}(\eta, 0)}{Z_{\text {pole }}+m_{\text {pole }}^{4} \int \frac{\mathrm{d} \eta}{\eta} \frac{1}{\eta^{2}} \rho_{\text {cut }}(\eta, 0)} .
$$

Equation (16) entails the information when the difference between pole and curvature masses grows large: First, decreasing $Z_{\text {pole }}$ increases the importance of the cut part, and, hence, the difference between curvature and pole mass grows. Second, if the spectral weight of $\rho_{\text {cut }}$ is taken at smaller spectral values, the integrals in (16) grow, and, hence, the difference between curvature and pole mass grows.

Translated back to Euclidean space-time, both processes lead to strong frequency and momentum dependences in the Euclidean propagator. In turn, if the pole term dominates the full spectral function, the full Euclidean propagator is well described by a propagator with a constant wave-function renormalization depicted in Fig. 4(c). A similar conclusion was also drawn in [64,72], where the relation between pole and curvature mass has also been investigated.

A very good estimate of the pole mass can be obtained from a Padé approximant of the propagator around the zero crossing of the real part of $\Gamma^{(2)}$ if the pole is sufficiently close to the Minkowski axis. This is certainly the case for the spectral functions depicted in Fig. 2. Our result for the different masses is shown together with the order parameter $\langle\sigma\rangle$ in Fig. 4(d). The order parameter shows a gentle decrease across the phase transition, signaling an extremely broad crossover.

For low temperatures, the masses stay roughly constant until the temperature scale is comparable to the lowest mas scale $2 \pi T \approx m_{\pi}$. In the intermediate regime, the temperature fluctuations become large enough to trigger the phase transition. The mass of the sigma meson can then also be used as an order parameter (see, e.g., [63]) and exhibits a clear minimum at the crossover. With an increasing temperature, all masses degenerate as they acquire a dominant thermal mass component $\sim T^{2}$, and the theory becomes effectively trivial.

Turning to the momentum dependence, the difference is negligibly small for the pion at very small temperatures, as expected since the momentum dependence of the two-point correlator shown in Fig. 4(a) is very close to unity. A small deviation can be seen around the phase transition, where the momentum dependence becomes maximal, hence, signaling the expected enhanced fluctuations for temperatures around the phase transition. The same behavior can be seen very well in the wave-function renormalizations [Fig. 4(c)] with a continuous temperature dependence. 


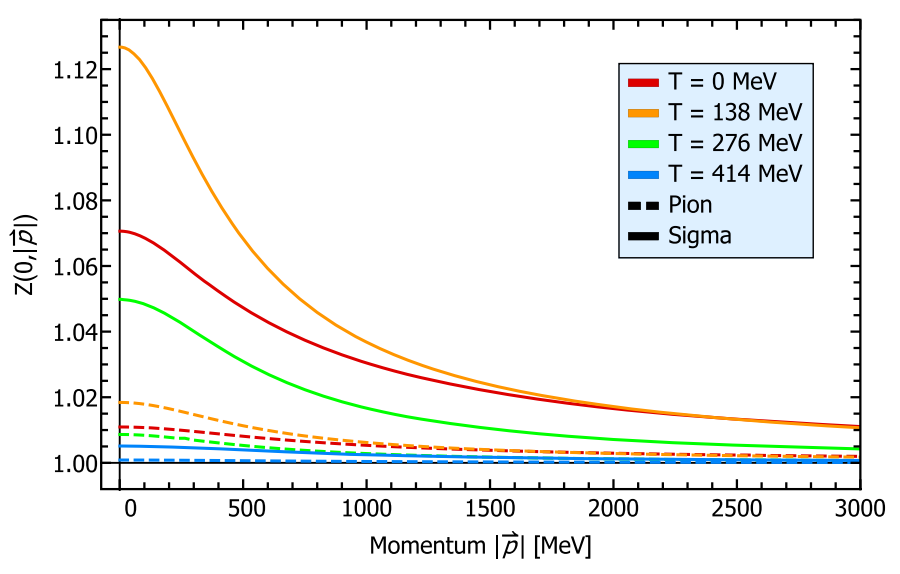

(a) Momentum-dependent Euclidean dressings.

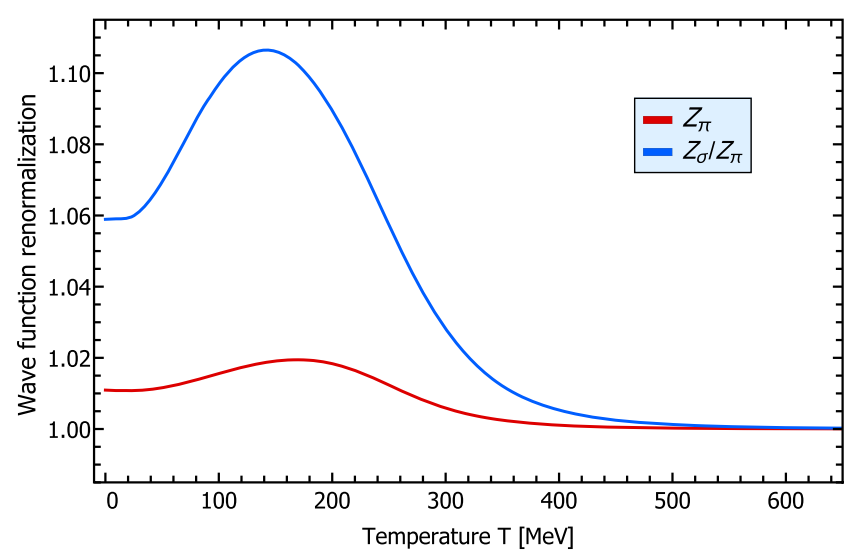

(c) Wave function renormalizations.

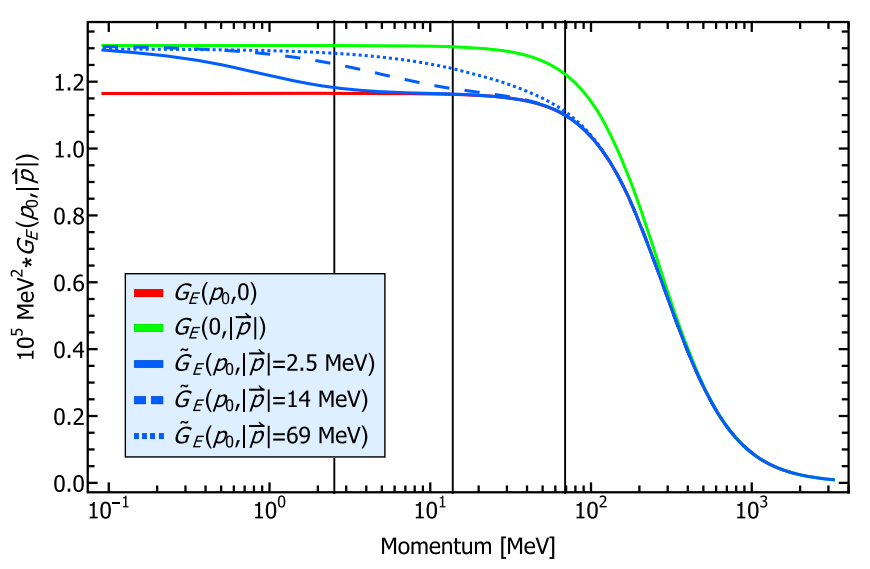

(b) Noncommuting limits (12) shown for the sigma propagator at $T=138 \mathrm{MeV}$.

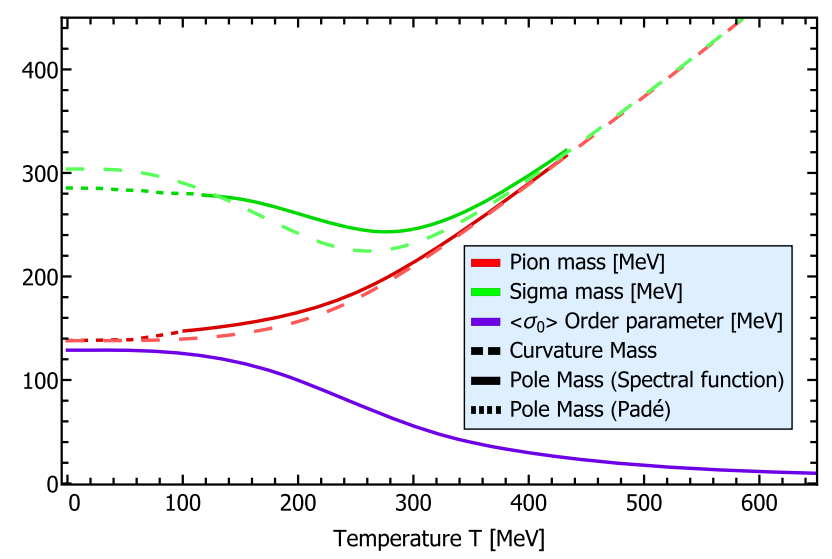

(d) Different masses and order parameter.

FIG. 4. Aspects concerning the relation between pole and curvature mass. In Fig. 4(a) the momentum dependence of the Euclidean dressing is shown for vanishing frequencies, the non-commuting nature thereof at finite Euclidean frequencies is depicted in Fig. 4(b). Additionally, the temperature dependence of the related wave function renormalization is shown in Fig. 4(c). The pole and curvature masses are compared in Fig. 4(d), where also the order parameter is shown in addition.

The more interesting case is the sigma, as its spectral function has no clear mass pole for low temperatures. The pole mass of the sigma mesons behaves more gently across the phase transition in comparison to the curvature mass, but it still exhibits a clear minimum at the crossover. The larger mismatch between the two masses can again be explained by the significantly stronger momentum dependence of the sigma meson at small and medium temperatures compared to the pion; cf. Fig. 4(a). In general, the qualitative strength of the mass difference can already be obtained from the temperature dependence of the constant wave-function renormalizations as $Z_{k}(0,|\vec{p}|) \approx Z_{k}(0, k)$.

Furthermore, one can use (9) in order to extract the pole mass from the two-point function. Combining the spectral representation (13) with (9), one obtains

$$
G_{E}(t, \vec{p})=\frac{1}{Z(0,0)} \int_{0}^{\infty} \mathrm{d} \eta e^{-\eta t} \rho(\eta, \vec{p}),
$$

i.e., the Fourier transformed propagator, which reduces to a calculation of a Laplace transform. In the case of a mass pole, we were able to extract the correct pole mass from (17) again; an example of the pion at a temperature $138 \mathrm{MeV}$ is shown in Fig. 5. Equation (17) allows us to calculate the contributions from different structures in the spectral function individually; i.e., we find the expected exponential decay (9) from the mass pole, and empirically, the contribution from the Landau cut is very well described by additionally introducing a quadratic time dependence in the exponent. Furthermore, their sum is already sufficient to describe the full time dependence at reasonable times; for extremely large times, the behavior is trivially dominated by the necessary numerical cut $\eta_{\min }$. Unfortunately, we were unable to extend this definition of the pole mass to the regime without a pole mass in the spectral function due to a combination of a lack of the functional form of the Landau cut and numerical uncertainties. 


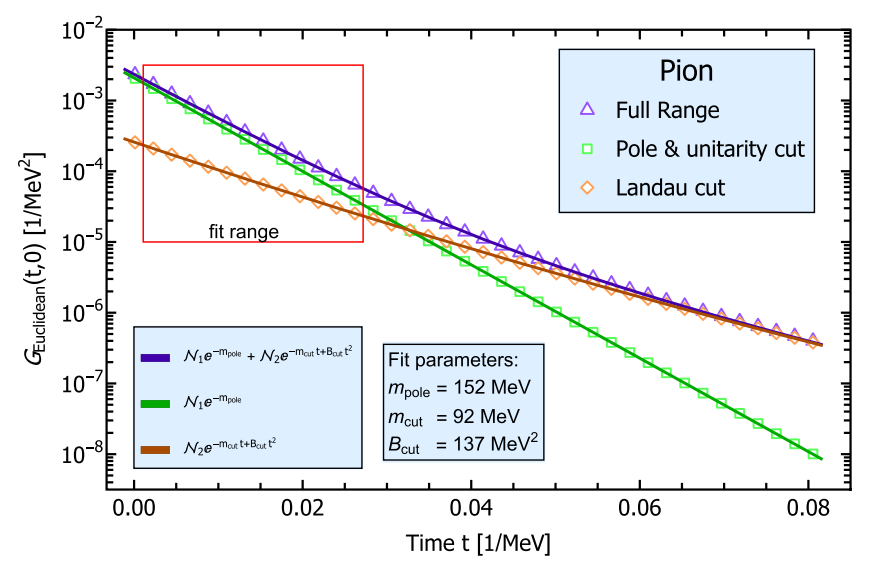

FIG. 5. Fourier transformed pion propagator.

\section{Spectral functions at finite external momentum}

Since there is only a very small difference between LPA and LPA' $+\mathrm{Y}$ in our current settings, the results with finite external momentum are calculated in LPA due to reduced numerical cost, due to which we also refrained from extrapolating our results to $\varepsilon \rightarrow 0$. The results are depicted in Fig. 6 for various temperatures and external momenta. The main differences between spectral functions at vanishing and finite external momentum are the uniform Lorentz boost of the mass pole and the unitarity cut, as well as the transport peak, at frequencies $\omega<|\vec{p}|$, arising from spacelike scattering processes at finite temperature and momenta with the heat bath; a detailed discussion about the involved kinematics can be found in [65]. Furthermore, the transport peak is, like the Landau cut, not Lorentz invariant, as it couples directly to the heat bath.

\section{Lorentz invariance}

A nontrivial consistency check of our results at finite external momenta is obtained by looking at the Lorentz invariance at a vanishing (or small) temperature. A Lorentzinvariant function must only depend on $p^{2}=\omega^{2}-\vec{p}^{2}$; i.e., for the spectral function, this translates to the property that $\rho\left(\sqrt{\omega^{2}+\vec{p}^{2}}, \vec{p}\right)$ must be independent of $\vec{p}$. Our results for the spectral functions at finite external momenta are depicted for this momentum configuration in Fig. 7 at a small temperature. The most notable breaking of Lorentz invariance is introduced by the finite value of the small parameter $\varepsilon$, but we also do not expect invariance for these parts of the spectral function. Additional breaking relative to the fixed heat bath is visible for small frequencies in the
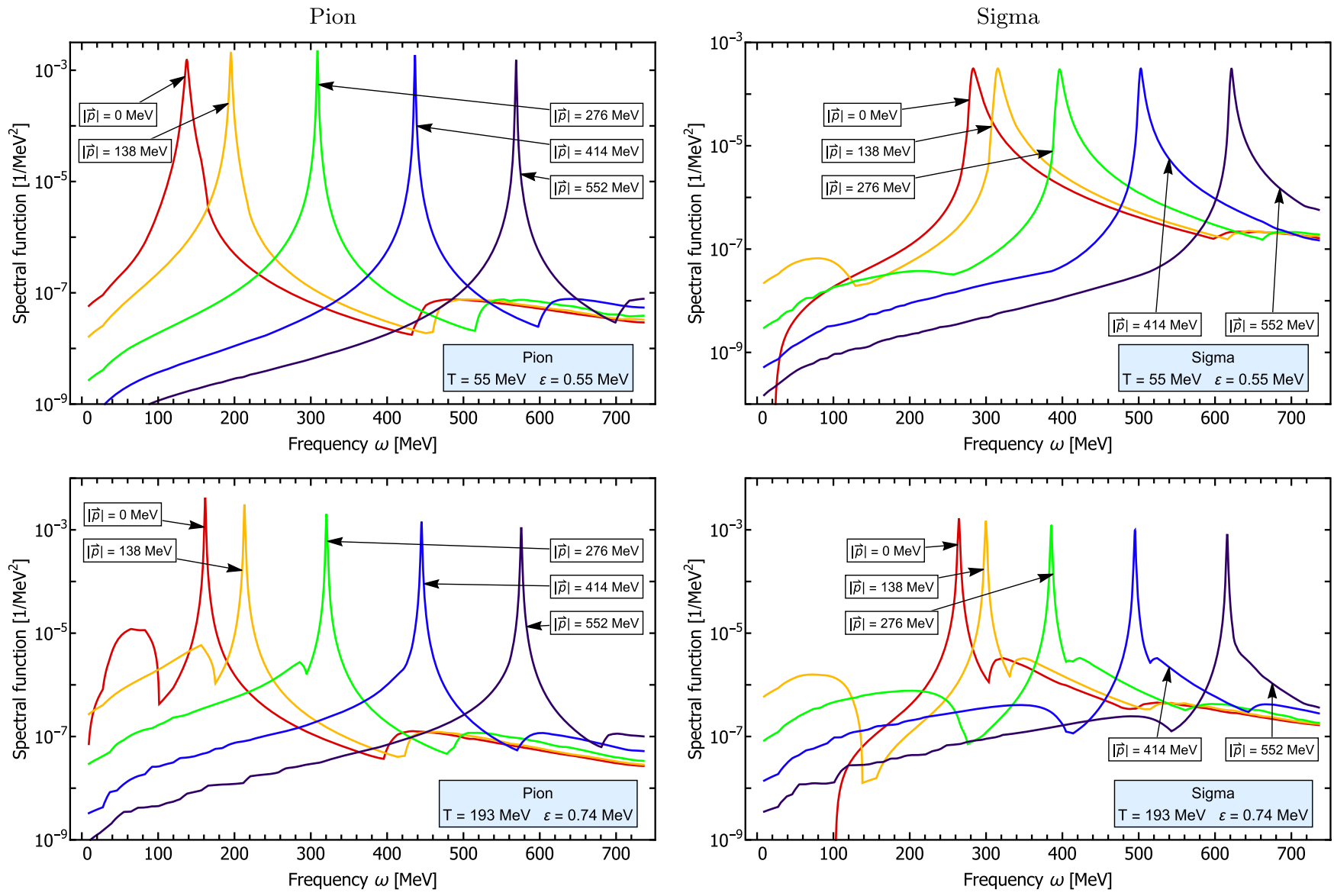

FIG. 6. Spectral functions at finite external momenta for different temperatures across the phase transition. 

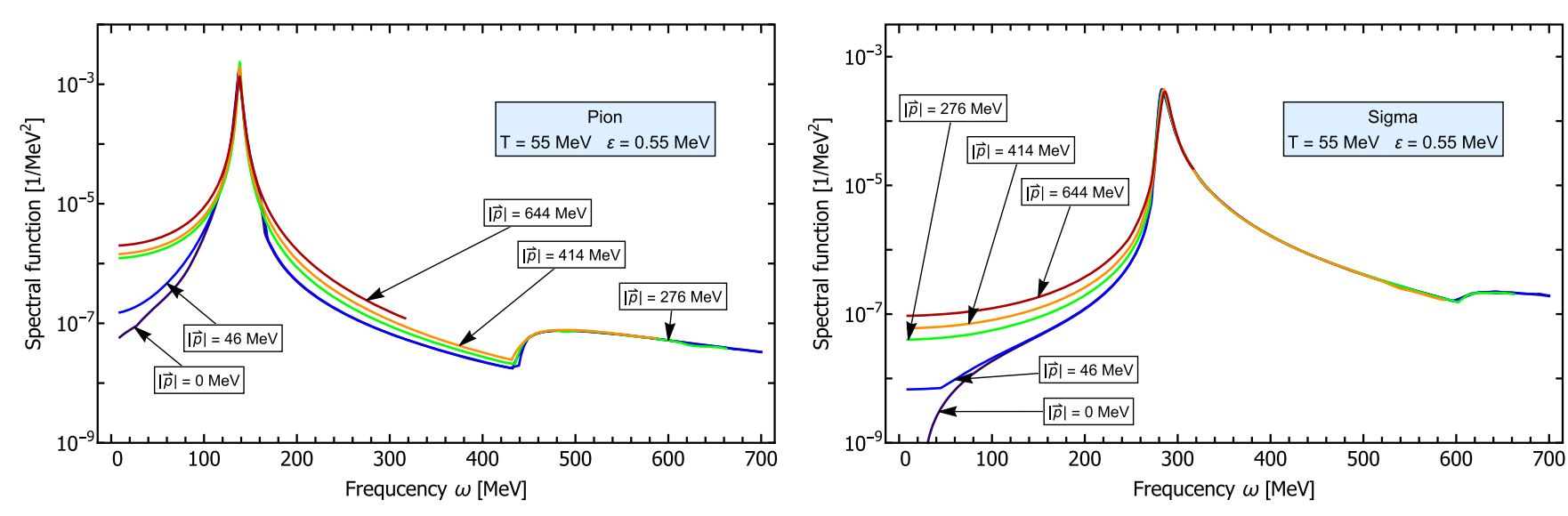

FIG. 7. Lorentz invariance of the spectral functions $\rho\left(\sqrt{\omega^{2}+\vec{p}^{2}}, \vec{p}\right)$ at a small temperature.

sigma spectral function and for frequencies around the mass pole in the pion spectral function due to the small temperature present; cf. the discussion in Secs. III A and IIIC. The remaining parts of the spectral functions, i.e., the position of the pole, the thresholds, and the continuum part, show perfect Lorentz invariance.

This is in contrast to most previous studies of spectral functions within the FRG where a regulator of the form (B1) was used and, therefore, Lorentz invariance explicitly broken $[14,65]$, and demonstrates one of the strengths of our approach.

\section{CONCLUSIONS}

In this work, we put forward a direct calculation of finite temperature spectral functions with the FRG in the $O(N)$ model. This direct computation is based on an $O(4) /$ Lorentz-invariant regularization scheme and can be performed on a fully numerical level, i.e., including the full momentum and frequency dependence of correlation functions. It demonstrates the applicability of the formalism put forward in [1] at finite temperatures.

The spectral functions for the pion and sigma meson are shown across the phase transition as a function of frequency and momentum. The four expected structures in the spectral functions, i.e., the mass pole, unitarity cut, Landau cut. and transport peak, are present and discussed in detail.

The spectra obtained allows us to investigate the important relation between the curvature and the pole mass. We find that the definition of the pole mass as inverse temporal length is accessible within our framework but unsuitable if particles are unstable and a pole is unidentifiable in the spectrum. An analytic relation between the pole and curvature mass is derived in the case of a stable particle and qualitatively verified that the difference is driven by nontrivial momentum dependences.

Furthermore, we explicitly verify the Lorentz invariance of the spectral function. Another major advantage of the employed framework is its numerical accessibility, which makes it easily usable in more complex theories. We hope to report on applications to Yang-Mills theory and QCD in the near future.

\section{ACKNOWLEDGMENTS}

We thank Anton K. Cyrol, Stefan Floerchinger, Fabian Rennecke, and Alexander Rothkopf for discussions. This work is supported by EMMI, Grant No. ERC-AdG-290623, the DFG through Grant No. STR1462/1-1, the BMBF Grant No. 05P12VHCTG, and is part of and supported by the DFG Collaborative Research Centre "SFB 1225 (ISOQUANT)." It is also supported in part by the Office of Nuclear Physics in the U.S. Department of Energy's Office of Science under Contract No. DE-AC02-05CH11231.

\section{APPENDIX A: REGULATOR AND CUTOFF SCALE}

In order to use a Lorentz-invariant regulator $R_{k}\left(p^{2}\right)$, we need to deal with additional poles/branch cuts necessarily introduced by the nontrivial analytic structure of $R_{k}\left(p^{2}\right)$. While the propagator $1 / \Gamma_{k}^{(2)}$ is restricted to have poles on the Minkowski axis, a Lorentz-invariant regulator with nontrivial momentum dependence that leaves all nonanalyticities of the regulated propagator $1 /\left(\Gamma_{k}^{(2)}(q)+R_{k}(q)\right)$ on the Minkowski axis is to date not known. As mentioned in Sec. II, we use the regulator introduced in [1], i.e.,

$$
R_{k}\left(p^{2}\right)=Z_{\sigma}\left[p^{2}+\Delta m_{r}^{2}\right] r_{k}\left(\frac{q^{2}+\Delta m_{r}^{2}}{k^{2}}\right) .
$$

For the shape function, we chose the exponential one

$$
r(x)=\frac{x^{m-1}}{e^{x^{m}}-1}
$$

with $m=2$. The masslike term $\Delta m_{r}^{2}$ has the effect of pushing regulator poles away from the Euclidean axis, 
TABLE I. Values for the parametrization of $\Delta m_{r}^{2}$.

\begin{tabular}{lcccc}
\hline \hline Parameter & $p_{0, \max }$ & $\alpha$ & $\beta$ & $n$ \\
\hline Value & $3.45 \mathrm{GeV}$ & 2.5 & 0.44 & 150 \\
\hline \hline
\end{tabular}

while only having a small impact on the analytic structure of the unregulated propagator. It is parametrized as a smooth theta function

$$
\Delta m_{r}^{2}=\alpha \frac{p_{0, \max ^{2}}}{1+\left(\frac{\beta k}{p_{0, \max }}\right)^{n}},
$$

where we have chosen our parameters according to Table I. These parameters are chosen such that the initial conditions $\Gamma_{\Lambda}$ are unchanged compared to the standard case $\Delta m_{r}^{2}=0$. In vacuum, it is possible to show for LPA that the regulator poles do not contribute to a certain frequency range if $p_{0, \max }$ is sufficiently large and the available frequency range corresponds roughly to its value. For finite temperature, this is not true anymore, as the correction factor for simple poles at position $z_{0}$ is proportional to $n_{B}\left(z_{0}+\mathrm{i} p_{0}\right)-n_{B}\left(z_{0}\right)$, which is only sufficiently suppressed for $\left|z_{0}\right| / T \gg 1$. Therefore, we have restricted our frequency range to $720 \mathrm{MeV}$, where we have checked for explicit independence of the results on the parametrization of $\Delta m_{r}^{2}$.

The large value of $\Delta m_{r}^{2}$, which enables us to resolve a large range of frequencies, comes with the downside that the cutoff scale $\Lambda_{\mathrm{UV}}$ must be sufficiently large. In order to still fulfill the requirement of unchanged initial conditions compared to a vanishing $\Delta m_{r}^{2}$, we have chosen $\Lambda_{\mathrm{UV}}=8.28 \mathrm{GeV}$; therefore, the interpretation of our results as an effective theory of low energy QCD is, strictly speaking, not possible. Nevertheless, our results demonstrate the applicability of the method to extract real-time correlation functions from the FRG via analytic continuation, and the qualitative features stay unchanged compared to the usual $O(N)$ model with a lower cutoff. We are unable to fix all values to their physical ones, as we observe a loss of solution similar to [73] restricting us from tuning to arbitrary IR values. Moreover, we also observed a smaller and smaller range of initial values that do not lead to a breakdown of the numerics as we increase our truncation. Therefore, we chose $f_{\pi} / m_{\pi}=0.93$ and $m_{\sigma} / m_{\pi}=2.09$, which fulfill the requirement $m_{\sigma} / m_{\pi}>2$, resulting in the sigma being an unstable particle. For all truncations, the initial values were chosen such that the curvature masses agree.

\section{APPENDIX B: DIFFERENT REGULATORS}

In addition to the regulator described in Appendix A, it is also insightful to compare to the Lorentz invariance breaking regulator (B1), which has been used in most FRG-related works so far,

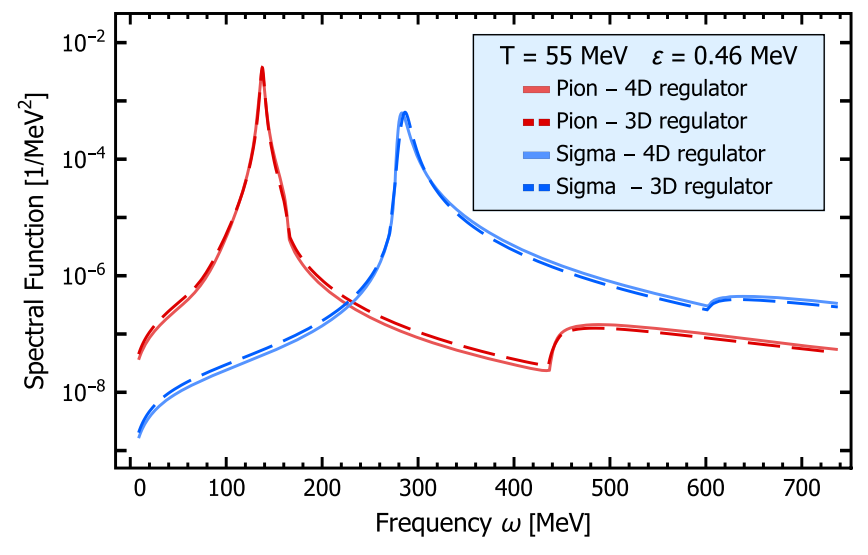

FIG. 8. Comparison of different regulators.

$$
R_{k}\left(\vec{p}^{2}\right)=\left(k^{2}-\vec{p}^{2}\right) \Theta\left(k^{2}-\vec{p}^{2}\right) .
$$

This regulator has the advantage that the Matsubara sum and the following analytic continuation can be performed analytically. Therefore, we compare our results obtained with the Lorentz-invariant regulator(A1) against the results obtained with (B1) for the LPA case and vanishing external momenta in Fig. 8. This shows that the difference between the two regulators is marginal, which can be explained by the small breaking of Lorentz invariance for $\vec{p}=0$ (cf. the discussion in Sec. III C) and the already very similar running of the Euclidean system for the two regulators [74] in this regime. For larger temperatures, the difference is dominantly driven by the difference in the condensate, leading to a general mismatch between the spectral function.

\section{APPENDIX C: NUMERICAL DETAILS}

We followed the general workflow outlined in [45]. The flow equations are derived using DoFun [75], traced and optimized using the FormTracer [76] utilizing FORM [77], and solved using the FRGSOLVER, a C++ framework developed and maintained by the fQCD Collaboration [62].

In order to implement the procedure outlined in Sec. II, the equations are solved as

$$
\begin{aligned}
& \Gamma^{(2)}(\omega, \vec{p}) \\
& =\Gamma_{\Lambda}^{(2)}(\omega, \vec{p})+\lim _{\varepsilon \rightarrow 0} \int_{\Lambda}^{k_{\mathrm{IR}}} \mathrm{d} k \int_{0}^{\infty} \mathrm{d}^{3} \vec{q}\left\{\sum_{q_{0}=2 \pi n T}+\text { Correction }\right\}
\end{aligned}
$$

Flow $\left[\Gamma_{k}^{(2)}\left(q_{0}, \vec{q}, p_{0}=-\mathrm{i}(\omega+\mathrm{i} \varepsilon), \vec{p}\right)\right]$,

where "Correction" refers to the correction of the occupation numbers arising implicitly from the Matsubara sum. From the study of the flow equation using a 3D regulator, it is well known $[1,78]$ that the equations reduce to delta functions for the imaginary part when the limit $\varepsilon \rightarrow 0$ is taken by means of the Sokhotski-Plemelj theorem. Because of our Lorentz-invariant regulator (A1), we are, however, not able to resolve the Matsubara sum analytically and must 
take the limit numerically, therefore, greatly increasing the numerical cost of the calculation as the delta functions (and derivatives thereof) have to be resolved numerically for very small values of $\varepsilon$. In practice, the limit is performed using Richardson extrapolation with several small values of $\varepsilon$, and the independence of the result for different sets of $\varepsilon$ 's has been checked explicitly. From the symmetries of the two-point correlator, it is known that the leading term in $\varepsilon$ of the imaginary part behaves as $\mathcal{O}\left(\varepsilon^{1}\right)$ and the real part behaves as $\mathcal{O}\left(\varepsilon^{2}\right)$, and the exponents in the extrapolation are chosen accordingly.

The correction term can, in principle, also take branch cuts into account, as described in Sec. II, and, therefore, allows for a self-consistent treatment. With the current methods, this is numerically insurmountable as it introduces a second competing limit for the branch cut integrals.
[1] J. M. Pawlowski and N. Strodthoff, Phys. Rev. D 92 , 094009 (2015).

[2] N. Strodthoff, Phys. Rev. D 95, 076002 (2017).

[3] C. Wetterich, Phys. Lett. B 301, 90 (1993).

[4] D. F. Litim and J. M. Pawlowski, arXiv:hep-th/9901063.

[5] J. Berges, N. Tetradis, and C. Wetterich, Phys. Rep. 363, 223 (2002).

[6] J. Polonyi, Central Eur. J. Phys. 1, 1 (2003).

[7] J. M. Pawlowski, Ann. Phys. (Amsterdam) 322, 2831 (2007).

[8] H. Gies, Lect. Notes Phys. 852, 287 (2012).

[9] B.-J. Schaefer and J. Wambach, Phys. Part. Nucl. 39, 1025 (2008).

[10] O. J. Rosten, Phys. Rep. 511, 177 (2012).

[11] J. Braun, J. Phys. G 39, 033001 (2012).

[12] L. von Smekal, Nucl. Phys. B, Proc. Suppl. 228, 179 (2012).

[13] S. Floerchinger, J. High Energy Phys. 05 (2012) 021.

[14] K. Kamikado, N. Strodthoff, L. von Smekal, and J. Wambach, Eur. Phys. J. C 74, 2806 (2014).

[15] S. G. Jakobs, V. Meden, and H. Schoeller, Phys. Rev. Lett. 99, 150603 (2007).

[16] R. Gezzi, T. Pruschke, and V. Meden, Phys. Rev. B 75, 045324 (2007).

[17] T. Gasenzer and J. M. Pawlowski, Phys. Lett. B 670, 135 (2008).

[18] C. Karrasch, R. Hedden, R. Peters, T. Pruschke, K. Schönhammer, and V. Meden, J. Phys. Condens. Matter 20, 345205 (2008).

[19] M. Pietroni, J. Cosmol. Astropart. Phys. 10 (2008) 036.

[20] J. Berges and G. Hoffmeister, Nucl. Phys. B813, 383 (2009).

[21] A. Sinner, N. Hasselmann, and P. Kopietz, Phys. Rev. Lett. 102, 120601 (2009).

[22] L. Canet, H. Chaté, B. Delamotte, and N. Wschebor, Phys. Rev. Lett. 104, 150601 (2010).

[23] L. Bartosch, H. Freire, J. J. R. Cardenas, and P. Kopietz, J. Phys. Condens. Matter 21, 305602 (2009).

[24] T. Gasenzer, S. Kessler, and J. M. Pawlowski, Eur. Phys. J. C 70, 423 (2010).

[25] C. Karrasch, M. Pletyukhov, L. Borda, and V. Meden, Phys. Rev. B 81, 125122 (2010).

[26] C. Karrasch, V. Meden, and K. Schönhammer, Phys. Rev. B 82, 125114 (2010).

[27] S. G. Jakobs, M. Pletyukhov, and H. Schoeller, Phys. Rev. B 81, 195109 (2010).
[28] J. Serreau, Phys. Rev. Lett. 107, 191103 (2011).

[29] O. Kashuba, D. M. Kennes, M. Pletyukhov, V. Meden, and H. Schoeller, Phys. Rev. B 88, 165133 (2013).

[30] S. Mathey, T. Gasenzer, and J. M. Pawlowski, Phys. Rev. A 92, 023635 (2015).

[31] L. M. Sieberer, S. D. Huber, E. Altman,, and S. Diehl, Phys. Rev. B 89, 134310 (2014).

[32] D. Mesterházy, J. H. Stockemer, L. F. Palhares, and J. Berges, Phys. Rev. B 88, 174301 (2013).

[33] S. Floerchinger, M. Garny, N. Tetradis, and U. A. Wiedemann, J. Cosmol. Astropart. Phys. 01 (2017) 048.

[34] T. Prokopec and G. Rigopoulos, J. Cosmol. Astropart. Phys. 08 (2018) 013.

[35] F. L. Buessen, D. Roscher, S. Diehl, and S. Trebst, Phys. Rev. B 97, 064415 (2018).

[36] U. Ellwanger, M. Hirsch, and A. Weber, Z. Phys. C 69, 687 (1996).

[37] B. Bergerhoff and C. Wetterich, Phys. Rev. D 57, 1591 (1998).

[38] C. Husemann and M. Salmhofer, Phys. Rev. B 79, 195125 (2009).

[39] J. P. Blaizot, R. Méndez Galain, and N. Wschebor, Phys. Lett. B 632, 571 (2006).

[40] J.-P. Blaizot, R. Méndez-Galain, and N. Wschebor, Phys. Rev. E 74, 051116 (2006).

[41] J.-P. Blaizot, R. Méndez-Galain, and N. Wschebor, Phys. Rev. E 74, 051117 (2006).

[42] F. Benitez, J.-P. Blaizot, H. Chaté, B. Delamotte, R. Méndez-Galain, and N. Wschebor, Phys. Rev. E 85, 026707 (2012).

[43] M. Mitter, J. M. Pawlowski, and N. Strodthoff, Phys. Rev. D 91, 054035 (2015).

[44] N. Christiansen, B. Knorr, J. Meibohm, J. M. Pawlowski, and M. Reichert, Phys. Rev. D 92, 121501 (2015).

[45] A. K. Cyrol, L. Fister, M. Mitter, J. M. Pawlowski, and N. Strodthoff, Phys. Rev. D 94, 054005 (2016).

[46] A. K. Cyrol, M. Mitter, J. M. Pawlowski, and N. Strodthoff, Phys. Rev. D 97, 054006 (2018).

[47] A. K. Cyrol, M. Mitter, J. M. Pawlowski, and N. Strodthoff, Phys. Rev. D 97, 054015 (2018).

[48] T. Denz, J. M. Pawlowski, and M. Reichert, Eur. Phys. J. C 78, 336 (2018).

[49] M. Jarrell and J. E. Gubernatis, Phys. Rep. 269, 133 (1996).

[50] M. Asakawa, T. Hatsuda, and Y. Nakahara, Prog. Part. Nucl. Phys. 46, 459 (2001). 
[51] J. Engels and O. Vogt, Nucl. Phys. B832, 538 (2010).

[52] J. Pawlowski and A. Rothkopf, Phys. Lett. B 778, 221 (2018).

[53] A. Rothkopf, Phys. Rev. D 95, 056016 (2017).

[54] E.-M. Ilgenfritz, J. M. Pawlowski, A. Rothkopf, and A. Trunin, Eur. Phys. J. C 78, 127 (2018).

[55] R. Schmidt and T. Enss, Phys. Rev. A 83, 063620 (2011).

[56] M. Haas, L. Fister, and J. M. Pawlowski, Phys. Rev. D 90, 091501 (2014).

[57] N. Christiansen, M. Haas, J. M. Pawlowski, and N. Strodthoff, Phys. Rev. Lett. 115, 112002 (2015).

[58] F. Rose, F. Léonard, and N. Dupuis, Phys. Rev. B 91, 224501 (2015).

[59] G. Cuniberti, E. De Micheli, and G. A. Viano, Commun. Math. Phys. 216, 59 (2001).

[60] Y. Burnier, M. Laine, and L. Mether, Eur. Phys. J. C 71, 1619 (2011).

[61] F. Ferrari, Nucl. Phys. B909, 880 (2016).

[62] J. Braun, L. Corell, A. K. Cyrol, W.-j. Fu, M. Leonhardt, M. Mitter, J. M. Pawlowski, M. Pospiech, F. Rennecke, N. Strodthoff, and N. Wink (fQCD Collaboration).

[63] J. M. Pawlowski and F. Rennecke, Phys. Rev. D 90, 076002 (2014).

[64] A. J. Helmboldt, J. M. Pawlowski, and N. Strodthoff, Phys. Rev. D 91, 054010 (2015).
[65] R.-A. Tripolt, L. von Smekal, and J. Wambach, Phys. Rev. D 90, 074031 (2014).

[66] H. A. Weldon, Phys. Rev. D 28, 2007 (1983).

[67] R.-A. Tripolt, N. Strodthoff, L. von Smekal, and J. Wambach, Phys. Rev. D 89, 034010 (2014).

[68] T. Yokota, T. Kunihiro, and K. Morita, Prog. Theor. Exp. Phys. 2016, 073D01 (2016).

[69] Z. Wang and P. Zhuang, Phys. Rev. D 96, 014006 (2017).

[70] T. Yokota, T. Kunihiro, and K. Morita, Phys. Rev. D 96, 074028 (2017).

[71] A. Das, Finite Temperature Field Theory, World Scientific Lecture Notes in Physics (World Scientific, Singapore, 1997).

[72] G. Markó, U. Reinosa, and Z. Szép, Phys. Rev. D 96, 036002 (2017).

[73] G. Markó, U. Reinosa, and Z. Szép, Nucl. Phys. B913, 405 (2016).

[74] J. M. Pawlowski, M. Scherzer, N. Strodthoff, and N. Wink (to be published).

[75] M. Q. Huber and J. Braun, Comput. Phys. Commun. 183, 1290 (2012).

[76] A. K. Cyrol, M. Mitter, and N. Strodthoff, Comput. Phys. Commun. 219C, 346 (2017).

[77] J. Kuipers, T. Ueda, J. A. M. Vermaseren, and J. Vollinga, Comput. Phys. Commun. 184, 1453 (2013).

[78] C. Jung, F. Rennecke, R.-A. Tripolt, L. von Smekal, and J. Wambach, Phys. Rev. D 95, 036020 (2017). 